\title{
Synthesis and cis/trans isomerism of $\mathrm{N}$-alkyl-1,3-oxathiolane-2-imines
}

\author{
Andrey K. Shirayev,* Igor K. Moiseev, and Sergey S. Karpeev \\ Department of Chemistry and Technology, State Technical University, Samara 443100, Russia \\ E-mail: orgchem@,samgtu.ru
}

Dedicated to Professor N. S. Zefirov

(received 29 Apr 05; accepted 05 Jul 05; published on the web 18 Jul 05)

\begin{abstract}
Reaction of 1-adamantanol with 2-hydroxythiocyanates 2a-d containing imide, ester or ether groups gives $N$-(1-adamantyl)-1,3-oxathiolane-2-imines 6a-d. Reaction of isoborneol leads to $N$ bornyl-1,3-oxathiolane-2-imines 6e,f. The barrier of rotation for the imine double bond is measured based on ${ }^{1} \mathrm{H}$ NMR spectra.
\end{abstract}

Keywords: $N$-Substituted 1,3-oxathiolane-2-imines, 2-hydroxythiocyanates, cis/trans-isomerism

\section{Introduction}

Compounds containing an imine group are increasingly important in organic synthesis ${ }^{1}$ and the mechanism of the cis/trans isomerization of imines is being studied in detail. ${ }^{2}$ The 1,3oxathiolane-2-imine structure includes an exocyclic imine group and can be used in organic synthesis for the preparation of biologically active compounds. 1,3-Oxathiolane-2-imines have been isolated as stable $\mathrm{N}$-acyl or $\mathrm{N}$-carbamoyl derivatives during the study of the conversion of oxiranes to thiiranes. ${ }^{3}$ Some of these derivatives are even biologically active compounds. ${ }^{4}$ Unlike the synthesis of $N$-aryl-1,3-oxathiolane-2-imines, ${ }^{5}$ the reaction of isothiocyanates with oxiranes catalyzed by Lewis acids cannot be used for preparing $N$-alkyl derivatives. The reaction of methyl isothiocyanate with 2-alkyl-oxiranes catalyzed by tetra- $n$-butylammonium bromide (Scheme 1) yields 2-alkyl-6,8,10-trimethyl-7,9-dithio-6,8,10-triaza-1-oxa-4-thiaspiro[4.5] decane. ${ }^{6}$ Synthetic routes for the preparation of $N$-alkyl-1,3-oxathiolane-2-imines (Scheme 1) include the following reactions: (i) reaction of benzylisothiocyanate with 2-methyloxirane catalyzed by the dimethyltin diiodide - HMPTA complex, ${ }^{5 \mathrm{c}}$ (ii) reaction of 2,2-di- $n$-butyl-1,3,2oxathiostannolane with alkyl isothiocyanates, ${ }^{7}$ (iii) reaction of $N, S, S^{\prime}$-trialkyl iminodithiocarbonates with lithium diethylamide and aldehydes or ketones. ${ }^{8}$ 
<smiles>[R]C1COC12N(C)C(=S)N(C)C(=S)N2C</smiles><smiles>CCCC[Sn]1(CCCC)OCCS1</smiles><smiles>[R]CSC(=[R])SC</smiles>

\section{Scheme 1}

To develop a convenient preparative method for $N$-alkyl-1,3-oxathiolane-2-imine, we have found the reaction ${ }^{9}$ of 2-hydroxythiocyanates 2 with 1-adamantyl or tert-butyl alcohols 3 (Scheme 2). The synthesis was successful because the alkylation of the thiocyano group was faster than the intramolecular cyclization of 2-hydroxythiocyanate. It was established that 1,3oxathiolane-2-imine does not react with tertiary cations, and less stable 3-acetylamino-, 3hydroxy-, and 3-carboxy-1-adamantyl cations do not give $N$-alkyl-1,3-oxathiolane-2-imines. ${ }^{9 a} \mathrm{~N}$ Alkyl-1,3-oxathiolane-2-imines 6 exist as a mixture of cis/trans-isomers around the imine double bond which was confirmed by ${ }^{1} \mathrm{H}$ NMR spectra. ${ }^{9 a}$ We describe here the preparation of $N$-alkyl1,3-oxathiolane-2-imines containing additional functional groups and the study of the cis/transisomerism of the $N$-1-adamantyl derivative.

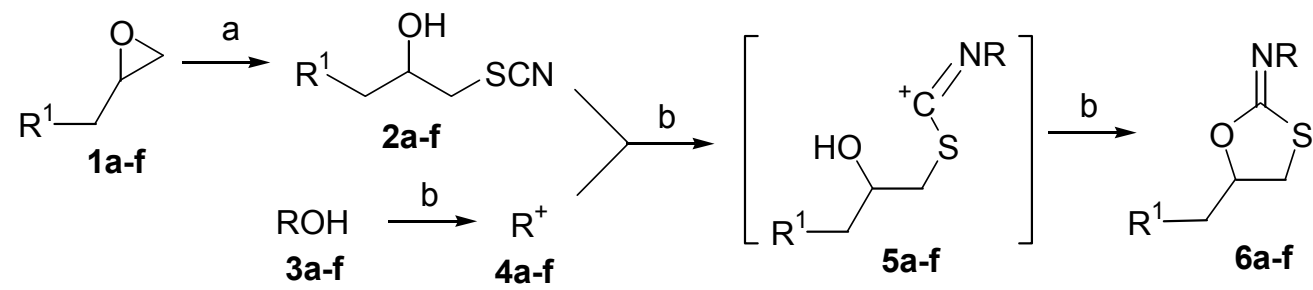

Scheme 2. For designation of $\mathrm{R}$ and $\mathrm{R}^{1}$ see Table 1. (a) $\mathrm{NH}_{4} \mathrm{SCN}$ (0.9 equivalent), $\mathrm{CH}_{3} \mathrm{COOH}$, 10-15 ${ }^{\circ} \mathrm{C}, 1-2$ h. (b) $\mathrm{ROH}$ (0.9 equivalent), $\mathrm{H}_{2} \mathrm{SO}_{4}-\mathrm{CH}_{3} \mathrm{COOH}, 0-10{ }^{\circ} \mathrm{C}, 1-2 \mathrm{~h}$. 
Table 1. Preparation of $N$-substituted 1,3-oxathiolane-2-imines 6a-f

\begin{tabular}{cccc}
\hline Entries & $\mathrm{R}^{1}$ & $\mathrm{R}$ & Yield, \% \\
\hline a & Phthalimido & 1-Adamantyl & 69 \\
b & PhCOO & 1-Adamantyl & 67 \\
c & 1-Adamantylacetoxy & 1-Adamantyl & 72 \\
d & $4-\mathrm{NO}_{2} \mathrm{C}_{6} \mathrm{H}_{4} \mathrm{O}$ & 1-Adamantyl & 56 \\
e & $\mathrm{H}$ & Isobornyl & 54 \\
f & $\mathrm{Cl}$ & Isobornyl & 48 \\
\hline
\end{tabular}

\section{Results and Discussion}

2-Hydroxythiocyanates react with tertiary alcohols in a mixture of sulfuric and acetic acids, which are to be neutralized in order to separate the products. These acidic conditions limit the substrates of the reaction, since the cations can lead to alkenes, polymerization products and rearrangements. Aryl groups can be involved in the alkylation by the cations. We have found that the compounds 1a-d containing phthalimido, ester and ether groups yield 5 -substituted $N$-(1adamantyl)-1,3-oxathiolane-2-imines 6a-d. The compounds 6a-d can be used to prepare 1,3oxathiolane alcohols and amines, which are interesting for searching biologically active compounds.

Isoborneol is the first secondary alcohol to be successfully used to prepare $N$-alkyl-1,3oxathiolane-2-imines 6e,f. Alkylation of 2-hydroxythiocyanate by isoborneol seems to be faster than the intramolecular cyclization. The first step of the process is the Ritter reaction employed for thiocyanates. ${ }^{10}$ But isoborneol can undergo the Wagner-Meerwein rearrangement in the course of the Ritter reaction. For example, the reaction of isoborneol (7) with chloroacetonitrile in sulfuric acid (Scheme 3) gives a 9:1 mixture of 3-exo-(chloroacetylamino)isocamphane (8) and 2-exo-(chloroacetylamino)bornane (9). ${ }^{11}$

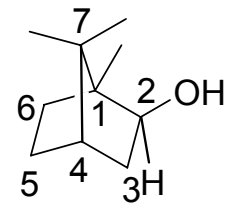

7

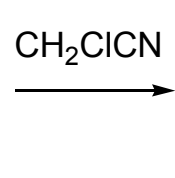

8

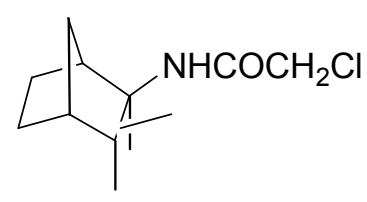

8

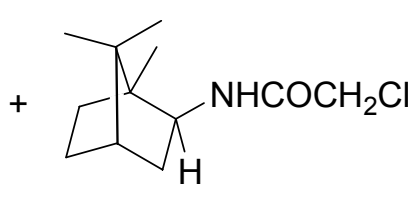

9

Scheme 3. Reaction of isoborneol with chloroacetonitrile. ${ }^{11}$

The reaction of isoborneol with 2-hydroxythiocyanates 2 e,f yields the bornyl derivatives 6e,f. The intermolecular reaction of the non-classical isobornyl cation with a thiocyano group seems to be faster than the rearrangement of this cation to the isocamphane structure. The 
structure of $\mathbf{6 e}, \mathbf{f}$ was confirmed by ${ }^{13} \mathrm{C}$ NMR spectra (Table 2). Signals of $\mathbf{6 e}$ and isoborneol are very close to each other, whereas the spectrum of the isocamphane derivative $\mathbf{8}$ is quite different from $6 \mathbf{e}$ and isoborneol. Signals of the carbon atoms located far from the polar substituent in $\mathbf{6 e}$ and isoborneol have almost the same values. The signals of the isocamphane methylene carbon atoms occur at high field, whereas the methyl signals occur at low field, which can be explained by the position of these atoms and the functional group in the bicyclic cage structure.

Table 2. ${ }^{13} \mathrm{C}$ NMR Signals for bicyclic fragment of $\mathbf{6 e}, \mathbf{6 f}$, isoborneol (7) and 3-exo(chloroacetylamino)isocamphane ${ }^{11}(\mathbf{8})$

\begin{tabular}{cc}
\hline Entries & ${ }^{13} \mathrm{C} \mathrm{NMR}$ \\
\hline $\mathbf{6 e}$ & $49.5,46.8\left(\mathrm{C}^{1}, \mathrm{C}^{7}\right) ; 71.9+72.3($ cis + trans $2-\mathrm{CHN}) ; 37.3,36.4,27.4$ \\
& $\left(3-\mathrm{CH}_{2}, 5-\mathrm{CH}_{2}, 6-\mathrm{CH}_{2}\right) ; 45.3(4-\mathrm{CH}) ; 20.4,20.3\left(7-\mathrm{CH}_{3}\right) ; 12.1\left(1-\mathrm{CH}_{3}\right)$ \\
$\mathbf{6 f}$ & $49.9,47.1\left(\mathrm{C}^{1}, \mathrm{C}^{7}\right) ; 72.6+72.7($ cis + trans $2-\mathrm{CHN}) ; 36.5,33.7,27.5$ \\
& $\left(3-\mathrm{CH}_{2}, 5-\mathrm{CH}_{2}, 6-\mathrm{CH}_{2}\right) ; 45.5(4-\mathrm{CH}) ; 20.6,20.4\left(7-\mathrm{CH}_{3}\right) ; 12.3\left(1-\mathrm{CH}_{3}\right)$ \\
7 & $49.1,46.5\left(\mathrm{C}^{1}, \mathrm{C}^{7}\right) ; 79.9(2-\mathrm{CHO}) ; 40.6,34.1,27.4\left(3-\mathrm{CH}_{2}, 5-\mathrm{CH}_{2}, 6-\mathrm{CH}_{2}\right) ;$ \\
& $45.3(4-\mathrm{CH}) ; 20.7,20.3\left(7-\mathrm{CH}_{3}\right) ; 11.5\left(1-\mathrm{CH}_{3}\right)$ \\
$\mathbf{8}$ & $50.2,48.9(1-\mathrm{CH}, 4-\mathrm{CH}) ; 44.6\left(\mathrm{C}^{2}\right) ; 64.5\left(\mathrm{C}^{3}\right) ; 22.9\left(5-\mathrm{CH}_{2}\right) ;$ \\
& $22.9\left(6-\mathrm{CH}_{2}\right) ; 34.5\left(7-\mathrm{CH}_{2}\right) ; 25.8,22.8\left(2-\mathrm{CH}_{3}\right) ; 17.3\left(3-\mathrm{CH}_{3}\right)$ \\
\hline
\end{tabular}

The bornyl $\mathrm{C}^{2}$ atom gives two NMR signals, which correspond to the cis/trans isomers. The imino carbon atom also gives two signals. To study the isomerism, ${ }^{1} \mathrm{H}$ NMR spectra were taken at temperatures $20-95{ }^{\circ} \mathrm{C}$ for $N$-(1-adamantyl)-1,3-oxathiolane-2-imine (6g). The multiplicity of the methylene heterocyclic protons disappears at $75{ }^{\circ} \mathrm{C}$ for $\mathrm{CH}_{2} \mathrm{~S}$ and at $85{ }^{\circ} \mathrm{C}$ for $\mathrm{CH}_{2} \mathrm{O}$ (Fig. 1). The energy characteristics of the isomerization were calculated using the Eyring equation for the rates extracted from the ${ }^{1} \mathrm{H}$ NMR spectra by the MEXICO line-shape analysis. ${ }^{12}$ The calculation of linear plot for $\ln (\mathrm{k} / \mathrm{T})-\ln (\mathrm{K} / \mathrm{h})$ vs $1000 / \mathrm{T}$ gave the following: slope=$7.32 \pm 0.15$, intercept $=-3.92 \pm 0.42$; where $\ln (\mathrm{K} / \mathrm{h})=23.76024,{ }^{12}$ slope $=\Delta H^{\ddagger} / \mathrm{R}$, and intercept $=\Delta S^{\neq}$ /R. The corresponding energies of activation are the following: $\Delta G_{298}^{\neq}=70.6 \pm 2.2 \mathrm{~kJ} / \mathrm{mol}, \Delta H^{\neq}=$ $60.9 \pm 1.2 \mathrm{~kJ} / \mathrm{mol}, \Delta S^{\neq}=-33 \pm 4 \mathrm{~J} /(\mathrm{mol} \mathrm{K})$. The close energy barriers have been found for $N$ substituted $2 H$-pyran-2-imines: $\Delta G_{T c}^{\neq}=73.5-80.7 \mathrm{~kJ} / \mathrm{mol}$ for $N$-phenyl derivatives, and $\Delta G_{T c}^{\neq}=$ 85.4-91.9 $\mathrm{kJ} / \mathrm{mol}$ for $N$-isopropyl derivative. ${ }^{13}$ The lower barrier for $N$-(1-adamantyl)-1,3oxathiolane-2-imine compared to $\mathrm{N}$-isopropyl-2H-pyran-2-imine can be explained by the conjugation of unshared electrons pairs of the two heteroatoms with the imine bond. 


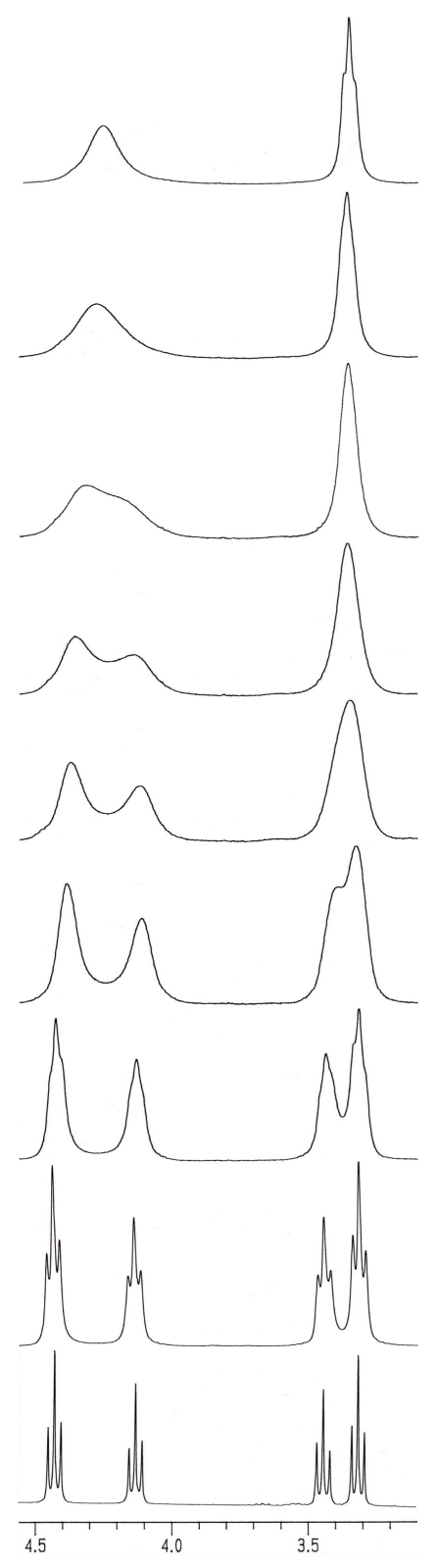

Figure 1. ${ }^{1} \mathrm{H}$ NMR spectra of $N$-(1-adamantyl)-1,3-oxathiolane-2-imine at 30, 50, 60, 70, 75, 80, $85,90,95^{\circ} \mathrm{C}$ starting from the bottom spectrum. 


\section{Conclusions}

The new route for the preparation of $N$-alkyl-1,3-oxathiolane-2-imines has been extended to oxiranes containing imide, ester and ether functional groups, and to isobornyl alcohols. The isobornyl cage does not undergo the isomerization during the reaction. Unfortunately, 2adamantanol did not yield any alkylated products because of the intramolecular cyclization of 2hydroxythiocyanate was faster than both the alkylation of the thiocyano group by the 2adamantyl cation and the isomerization of the latter to the 1-adamantyl cation. cis/transIsomerism of $\mathrm{N}$-alkyl-1,3-oxathiolane-2-imines has been confirmed by ${ }^{13} \mathrm{C} \mathrm{NMR}$ data and the energy barrier of the rotation around the $\mathrm{C}=\mathrm{N}$ bond has been calculated from the temperature dependence of ${ }^{1} \mathrm{H}$ NMR spectra.

\section{Experimental Section}

General Procedures. Melting points were determined on a hot-stage apparatus and are uncorrected. IR spectra were recorded on a Shimadzu FTIR-8500S spectrometer, mass spectra were recorded using DEP mode on Finnigan DSQ GC-MS with an ionization potential of $70 \mathrm{ev,}$ ${ }^{1} \mathrm{H}$ and ${ }^{13} \mathrm{C}$ NMR spectra were recorded in $\mathrm{CDCl}_{3}$ with TMS as the internal standard for ${ }^{1} \mathrm{H}$ $(90 \mathrm{MHz})$ and ${ }^{13} \mathrm{C}(22 \mathrm{MHz}) .{ }^{1} \mathrm{H}$ NMR spectra at temperatures $20-100{ }^{\circ} \mathrm{C}$ were recorded in DMSO-d $_{6}$ for ${ }^{1} \mathrm{H}(300 \mathrm{MHz})$.

Materials. The oxiranes 1a-d were prepared according to the following procedures mentioned in literature: $^{14}$ N-glycidyl phthalimide (1a) ${ }^{14 a}$ glycidyl benzoate (1b) ${ }^{14 a}$ glycidyl 1adamantylacetate (1c); ${ }^{14 \mathrm{~b}}$ glycidyl 4-nitrophenyl ether (1d). ${ }^{14 \mathrm{a}}$

\section{General procedure for the preparation of 6a-d,g}

A solution of ammonium thiocyanate $(5 \mathrm{~g}, 0.065 \mathrm{~mol})$ and oxirane 1a-d $(0.08 \mathrm{~mol})$ in glacial acetic acid $(15 \mathrm{ml}, 0.25 \mathrm{~mol})$ was stirred for $1-2 \mathrm{~h}$. The acetic acid solution was added dropwise to a solution of 1-adamantanol $(8 \mathrm{~g}, 0.053 \mathrm{~mol})$ in sulfuric acid $(30 \mathrm{ml}, \mathrm{d} 1.84)$ at $5-10{ }^{\circ} \mathrm{C}$. The reaction mixture was poured on ice followed by extraction with chloroform and neutralization with sodium carbonate. The crude product was extracted with methylene chloride, filtered through a silica gel and the solvent was distilled off.

\section{General procedure for the preparation of $6 \mathrm{e}-\mathrm{f}$}

A solution of ammonium thiocyanate $(5 \mathrm{~g}, 0.065 \mathrm{~mol})$, oxirane $1 \mathrm{e}-\mathrm{f}(0.08 \mathrm{~mol})$ and isoborneol $(8 \mathrm{~g}, 0.053 \mathrm{~mol})$ in glacial acetic acid $(15 \mathrm{ml}, 0.25 \mathrm{~mol})$ was stirred for $1-2 \mathrm{~h}$. The acetic acid solution was added dropwise to sulfuric acid $(30 \mathrm{ml}, \mathrm{d} 1.84)$ at 5-10 oC. The following work up was the same as for $6 a-d$. 
N-(1-Adamantyl)-5-phthalimidomethyl-1,3-oxathiolane-2-imine (6a). White microcrystals from acetone (69\%), $\mathrm{mp} 145-147^{\circ} \mathrm{C}$; IR $\left(\mathrm{KBr} / \mathrm{cm}^{-1}\right)$ 1772, 1720, 1710, 1657, 1466, 1421, 1396, 1057, 1034, 721; ${ }^{1} \mathrm{H}$ NMR $\delta 1.70(\mathrm{~m}, 6 \mathrm{H}), 1.99(\mathrm{~m}, 6 \mathrm{H}), 2.18(\mathrm{~m}, 3 \mathrm{H}), 3.40(\mathrm{dd}, J=5.4 \mathrm{~Hz}, J=$ $13.5 \mathrm{~Hz}, 2 \mathrm{H}), 4.08(\mathrm{dd}, J=4.6 \mathrm{~Hz}, J=13.0 \mathrm{~Hz}, 2 \mathrm{H}), 4.95(\mathrm{~m}, 1 \mathrm{H}), 7.90(\mathrm{~m}, 4 \mathrm{H}) ;{ }^{13} \mathrm{C}$ NMR $\delta$ 29.1, $32.4\left(\mathrm{CH}_{2} \mathrm{~S}\right), 35.8,39.3\left(\mathrm{CH}_{2} \mathrm{~N}\right), 41.1+41.8,52.6+56.1,74.0+79.9(\mathrm{CHO}), 122.8,131.2$, 133.8, 152.6+155.1 (C=N), $167.2(\mathrm{C}=\mathrm{O}) ; m / z: 396\left(\mathrm{M}^{+}, 11 \%\right), 219(75 \%), 186(100 \%), 175$ (29\%), 160 (45\%), 135 (24\%), 120 (15). Anal. Calcd for $\mathrm{C}_{22} \mathrm{H}_{24} \mathrm{~N}_{2} \mathrm{O}_{3} \mathrm{~S}: \mathrm{C}, 66.64 ; \mathrm{H}, 6.10 ; \mathrm{N}$, 7.07. Found: C, 66.50; H, 5.98; N, 7.23.

$\mathrm{N}$-(1-Adamantyl)-5-benzoyloxymethyl-1,3-oxathiolane-2-imine (6b). White microcrystals

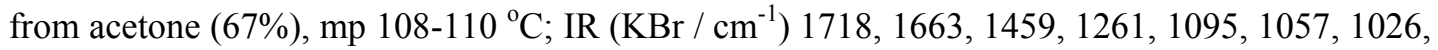
716; ${ }^{1} \mathrm{H}$ NMR $\delta 1.68(\mathrm{~m}, 6 \mathrm{H}), 1.97(\mathrm{~m}, 6 \mathrm{H}), 2.15(\mathrm{~m}, 3 \mathrm{H}), 3.4(\mathrm{~m}, 2 \mathrm{H}), 4.4(\mathrm{~m}, 2 \mathrm{H}), 4.55+4.84$ $(\mathrm{m}, 1 \mathrm{H}), 7.45(\mathrm{~m}, 2 \mathrm{H}), 7.56(\mathrm{~m}, 1 \mathrm{H}), 8.07(\mathrm{~m}, 2 \mathrm{H}) ;{ }^{13} \mathrm{C}$ NMR $\delta 29.7+29.9,31.7+34.9\left(\mathrm{CH}_{2} \mathrm{~S}\right)$, 36.5+36.6, 41.8+42.5, 53.3+56.7 (C-N), 64.1+64.2 $\left(\mathrm{CH}_{2} \mathrm{O}\right), 75.2+80.4(\mathrm{CHO}), 128.5,129.4$, 129.9, 133.5, 153.4+155.4 (C=N), $166.2(\mathrm{C}=\mathrm{O})$; $\mathrm{MS} m / z: 371\left(\mathrm{M}^{+}, 12 \%\right), 194(91 \%), 177(12 \%)$, 161 (25\%), 135 (35\%), $120(30 \%), 105(100 \%), 93$ (17\%), 77 (38\%), 72 (60\%). Anal. Calcd for $\mathrm{C}_{21} \mathrm{H}_{25} \mathrm{NO}_{3} \mathrm{~S}: \mathrm{C}, 67.89 ; \mathrm{H}, 6.78 ; \mathrm{N}, 3.77$. Found: C, 67.77; H, 6.49; N, 3.68.

N-(1-Adamantyl)-5-(1-adamantyl)acetoxymethyl-1,3-oxathiolane-2-imine (6c). White microcrystals from pentane $(72 \%), \mathrm{mp} 84-88{ }^{\circ} \mathrm{C}$; IR $\left(\mathrm{KBr} / \mathrm{cm}^{-1}\right) 1734,1664,1452$, 1138, 1055; ${ }^{1} \mathrm{H}$ NMR $\delta{ }^{1} \mathrm{H}: 1.62(\mathrm{~m}, 21 \mathrm{H}), 2.0(\mathrm{~m}, 11 \mathrm{H}), 3.3(\mathrm{~m}, 2 \mathrm{H}), 4.3(\mathrm{~m}, 2 \mathrm{H}), 4.52+4.82$ $(\mathrm{m}, 1 \mathrm{H}) ;{ }^{13} \mathrm{C}$ NMR $\delta 28.6+29.7+29.9,32.9+34.8\left(\mathrm{CH}_{2} \mathrm{~S}\right), 36.5+36.6+36.8,41.8+42.4+42.5$, 48.6+48.7, 53.2+55.5 (C-N), 62.9+63.2 $\left(\mathrm{CH}_{2} \mathrm{O}\right), 75.1+80.7(\mathrm{CHO}), 153.1(\mathrm{C}=\mathrm{N}), 171.2(\mathrm{C}=\mathrm{O})$; m/z: $443\left(\mathrm{M}^{+}, 2 \%\right), 266(9 \%), 234$ (9\%), $210(9 \%), 193(30 \%), 177(35 \%), 149(11 \%), 135$ (100\%), 120 (19\%), 93 (21\%), 79 (21\%), 73 (30\%), 72 (50\%). Anal. Calcd for $\mathrm{C}_{26} \mathrm{H}_{37} \mathrm{NO}_{3} \mathrm{~S}: \mathrm{C}$, 70.39; H, 8.41; N, 3.16. Found: C, 69.97; H, 8.37; N, 3.03.

$\mathrm{N}$-(1-Adamantyl)-5-(4-nitrophenoxy)methyl-1,3-oxathiolane-2-imine (6d). White microcrystals from acetone $(56 \%), \mathrm{mp} 165-167^{\circ} \mathrm{C}$; IR $\left(\mathrm{KBr} / \mathrm{cm}^{-1}\right) 1655,1591,1508,1340,1263,1254,1051$, 1036, 851, 752; ${ }^{1} \mathrm{H}$ NMR $\delta 1.63(\mathrm{~m}, 6 \mathrm{H}), 1.88(\mathrm{~m}, 6 \mathrm{H}), 2.05(\mathrm{~m}, 3 \mathrm{H}), 3.48(\mathrm{dd}, J=9.0 \mathrm{~Hz}, J=$ $8.0 \mathrm{~Hz}, 1.3 \mathrm{H}), 3.58(\mathrm{dd}, J=9.0 \mathrm{~Hz}, J=7.0 \mathrm{~Hz}, 0.7 \mathrm{H}), 4.27(\mathrm{~m}, 2 \mathrm{H}), 4.73+5.07(\mathrm{~m}, 1 \mathrm{H}), 6.96(\mathrm{~d}$, $J=9.0 \mathrm{~Hz}, 2 \mathrm{H}), 8.19(\mathrm{~d}, J=9.0 \mathrm{~Hz}, 2 \mathrm{H}) ;{ }^{13} \mathrm{C} \mathrm{NMR} \delta 29.7+29.8,35.0\left(\mathrm{CH}_{2} \mathrm{~S}\right), 36.5,41.8+42.5$, $53.4(\mathrm{C}-\mathrm{N}), 67.7\left(\mathrm{CH}_{2} \mathrm{O}\right), 74.7(\mathrm{CHO}), 114.6,126.0,142.1,162.6(\mathrm{C}=\mathrm{N}) ; \mathrm{m} / z: 388\left(\mathrm{M}^{+}, 9 \%\right)$, 331 (12\%), 211 (17\%), 149 (7\%), 135 (38\%), 93 (15\%), 79 (15\%), 73 (100\%). Anal. Calcd for $\mathrm{C}_{20} \mathrm{H}_{24} \mathrm{~N}_{2} \mathrm{O}_{4} \mathrm{~S}: \mathrm{C}, 61.84 ; \mathrm{H}, 6.23 ; \mathrm{N}, 7.21$. Found: C, 61.39; H, 6.41; N, 7.40.

$\mathbf{N}$-(2-exo-Bornyl)-5-methyl-1,3-oxathiolane-2-imine (6e). White microcrystals from pentane (54\%), mp 67-69 ${ }^{\circ} \mathrm{C}$; IR (KBr / cm $\left.{ }^{-1}\right) 1664,1452,1366,1167,1155,1090,1068,1024 ;{ }^{1} \mathrm{H}$ NMR $\delta 0.84(\mathrm{~s}, 3 \mathrm{H}), 0.87(\mathrm{~s}, 6 \mathrm{H}), 1.08(\mathrm{~s}, 3 \mathrm{H}), 1.15-1.80(\mathrm{~m}, 8 \mathrm{H}), 3.0(\mathrm{~m}, 1 \mathrm{H}), 3.4(\mathrm{~m}, 1 \mathrm{H}), 4.55(\mathrm{~m}$, $1 \mathrm{H}) ;{ }^{13} \mathrm{C}$ NMR $\delta 12.1\left(1-\mathrm{CH}_{3}\right), 18.9+19.0,20.3+20.4\left(7-\mathrm{CH}_{3}\right), 27.4,36.4,37.3,38.9\left(\mathrm{CH}_{2} \mathrm{~S}\right)$, 45.3, 46.8, 49.5, 71.9+72.3, 76.7, 157.0+159.0; m/z: $253\left(\mathrm{M}^{+}, 3 \%\right), 238(3 \%), 211(18 \%), 179$ (6\%), 151 (88\%), 136 (30\%), 118 (100\%), 108 (30\%), 102 (65\%), 95 (38\%), 93 (34\%), 81 (27\%), 75 (28\%), 74 (26\%), 67 (30\%). Anal. Calcd for $\mathrm{C}_{14} \mathrm{H}_{23} \mathrm{NOS}$ : C, 66.36; H, 9.15; N, 5.53. Found: C, 66.20; H, 8.85; N, 5.41. 
N-(2-exo-Bornyl)-5-chloromethyl-1,3-oxathiolane-2-imine (6f). White microcrystals from pentane (48\%), mp 117-119 ${ }^{\circ} \mathrm{C}$; IR $\left(\mathrm{KBr} / \mathrm{cm}^{-1}\right) 1659,1450,1149,1111,1084,1047,901 ;{ }^{1} \mathrm{H}$ NMR $\delta 0.81(\mathrm{~s}, 6 \mathrm{H}), 0.99(\mathrm{~s}, 3 \mathrm{H}), 1.69(\mathrm{~m}, 6 \mathrm{H}), 2.16(\mathrm{~s}, 1 \mathrm{H}), 2.83(\mathrm{~m}, 1 \mathrm{H}), 3.3(\mathrm{~m}, 2 \mathrm{H}), 3.6(\mathrm{~m}$, $2 \mathrm{H}), 4.63(\mathrm{~m}, 1 \mathrm{H}) ;{ }^{13} \mathrm{C}$ NMR $\delta 12.3\left(1-\mathrm{CH}_{3}\right), 20.4+20.6\left(7-\mathrm{CH}_{3}\right), 27.5,33.7,36.5,39.0\left(\mathrm{CH}_{2} \mathrm{~S}\right)$, $42.9\left(\mathrm{CH}_{2} \mathrm{Cl}\right), 45.5,47.1,49.9,72.6+72.7,78.3,157.7 ; \mathrm{m} / \mathrm{z}: 287\left(\mathrm{M}^{+}, 1 \%\right), 272(2 \%), 252(12 \%)$, 211 (17\%), 180 (17\%), 179 (24\%), 178 (27\%), 177 (30\%), 164 (15\%), $152(100 \%), 142$ (79\%), 136 (38\%), 109 (28\%), 108 (40\%), 95 (52\%), 93 (58\%), 81 (27\%), 67 (38\%). Anal. Calcd for $\mathrm{C}_{14} \mathrm{H}_{22}$ ClNOS: C, 58.42; H, 7.70; N, 4.87. Found: C, 58.32; H, 7.87; N, 4.54.

$\mathrm{N}$-(1-Adamantyl)-1,3-oxathiolane-2-imine (6g). White microcrystals from pentane (87\%), $\mathrm{mp}$ 85-86 ${ }^{\circ} \mathrm{C}$; IR $\left(\mathrm{KBr} / \mathrm{cm}^{-1}\right)$ 1657, 1450, 1119, 1094, 1055, 1034; ${ }^{1} \mathrm{H}$ NMR $\delta 1.68(\mathrm{~m}, 6 \mathrm{H}), 1.91$ $(\mathrm{m}, 6 \mathrm{H}), 2.11(\mathrm{~m}, 3 \mathrm{H}), 3.27(\mathrm{t}, J=6.6 \mathrm{~Hz}, 1 \mathrm{H}), 3.40(\mathrm{t}, J=6.6 \mathrm{~Hz}, 1 \mathrm{H}), 4.22(\mathrm{t}, J=6.6 \mathrm{~Hz}, 1 \mathrm{H})$, $4.46(\mathrm{t}, J=6.6 \mathrm{~Hz}, 1 \mathrm{H}) ;{ }^{13} \mathrm{C} \mathrm{NMR} \delta 29.5+29.6,30.6+33.3\left(\mathrm{CH}_{2} \mathrm{~S}\right), 36.3+36.4,41.5+42.4$, $52.8+56.2,65.9+71.6\left(\mathrm{CH}_{2} \mathrm{O}\right), 154.0+157.0(\mathrm{C}=\mathrm{N}) ; \mathrm{m} / \mathrm{z}: 237\left(\mathrm{M}^{+}, 24 \%\right), 180(33 \%), 135$ (100\%), 120 (19\%), $107(21 \%), 95$ (36\%), 93 (34\%), 79 (39\%), 60 (53\%). Anal. Calcd for $\mathrm{C}_{13} \mathrm{H}_{19} \mathrm{NOS}$ : C, 65.72; H, 8.07; N, 5.90. Found: C, 65.78; H, 8.05; N, 5.69

\section{Acknowledgments}

The authors thank Dr. Alexander Paptchikhine (Pharmacie, Sweden) for his help with the spectral research.

\section{References}

1. For examples see (a) Borg, G.; Chino, M.; Ellman, J. A. Tetrahedron Lett. 2001, 42, 1433. (b) Sharpless, K. B. Org. Lett. 1999, 1, 783. (c) Alvaro, G; Martelli, G.; Savoia, D.; Zoffoli, A. Synthesis 1998, 1773.

2. Johnson, J. E.; Morales, N. M.; Gorszyca, A. M.; Dolliver, D. D.; McAlister, M. A. J. Org. Chem. 2001, 66, 7979.

3. (a) Sergeev, P. G.; Kolychev, B. S. J. Gen. Chem. (USSR) 1937, 7, 1390. (b) Van Tamelen, E.E. J. Am. Chem. Soc. 1951, 73, 3444. (c) Price, C.C.; Kirk, P.F. J. Am. Chem. Soc. 1953, 75, 2396. (d) Wagner-Jauregg, T.; Häring, M. Helv. Chim. Acta 1958, 41, 377.

4. (a) Addor, R. W. U.S. Patent, 1966, 3, 281, 430; Chem. Abstr. 1967, 66, 65483s. (b) Feinauer, R.; Hamann, K. Ger. Patent, 1967, 1, 242, 631; Chem. Abstr. 1968, 68, 21923x. (c) Irikura, T.; Suzue, S.; Hasegawa, Y.; Kawasaki, H. Jap. Patent, 1971, 7, 105, 698; Chem. Abstr. 1971, 74, 141740f. (d) Pflugfelder, B., U.S. Patent, 1972, 3639 610; Chem. Abstr. 1972, 76, 140770v. (e) Oyamada, K.; Nagano, M.; Tobizuka, J.; Matsui, K. Jap. Patent, 1972, 7, 246, 064; Chem. Abstr. 1973, 78, 124598f. (f) Sauer, W.; Goldner, H.; Carstens, E. 
Ger. (East) Patent, 1974, 105, 215; Chem. Abstr. 1974, 81, 151824v. (g) Bollinger, F. G. Eur. Pat. Appl. 1980, EP 11473; Chem. Abstr. 1981, 94, 175100a.

5. (a) Ansari, M. H.; Ahmad, M. J. Chem. Res. (S) 1990, 199; J. Chem. Res. (M) 1990, 1733.

(b) Baba, A.; Shibata, I.; Kashiwagi, H.; Matsuda H. Bull. Chem. Soc. Jap. 1986, 59, 341.

(c) Shibata, I.; Baba, A.; Iwasaki, H.; Matsuda H. J. Org. Chem. 1986, 51, 2177. (d) Yano, K.; Amishiro, N.; Baba, A.; Matsuda, H. Bull. Chem. Soc. Jap. 1991, 64, 2661.

6. (a) Etlis, V. S.; Sineokov, A. P.; Razuvayev, G. A. Izv. AN USSR. Ser. Khim. 1964, 737. (b) Etlis, V. S.; Sineokov, A. P.; Razuvayev, G. A. Izv. AN USSR. Ser. Khim. 1964, 2051.

7. Sakai, S.; Niimi, H.; Kobayashi, T.; Ishii, Y. Bull. Chem. Soc. Jap. 1977, 50, 3271.

8. (a) Hoppe, D.; Follmann, R. Angew. Chem. 1977, 89, 478. (b) Hoppe, D.; Follmann, R. Liebigs Ann. Chem. 1980, 1779.

9. (a) Shirayev, A. K.; Moiseev, I. K.; Popov V. A. Zh. Org. Khim. 1992, 28, 418. (b) Shirayev, A. K.; Kong Thoo lin, P.; Moiseev, I. K. Synthesis 1997, 38.

10. (a) Riemschneider, R. J. Am. Chem. Soc. 1956, 78, 844. (b) Klimochkin, Y. N.; Moiseev, I. K. Zh. Org. Khim. 1987, 23, 2026. (c) Klimochkin, Y. N.; Moiseev, I. K.; Abramov, O. V.; Vladyko, G. V.; Korobchenko, L. V.; Boreko, E. I. Khim.-Pharm. Zh. 1991, 25, 49.

11. Lysenkov, V. I. Zh. Org. Khim. 1989, 25, 1570.

12. Bain, A. D. Prog. Nucl. Magn. Reson. Spectrosc. 2003, 43, 63.

13. Uncuta, C.; Tudose, A.; Caproiu, M. T.; Udrea, S. ARKIVOC 2003, (i), 29.

14. (a) Paken, A. M. Epoxy compounds and epoxy resins, Leningrad, Goschimizdat 1962. (b) Shirayev, A. K.; Moiseev, I. K.; Stroganov, V. F. Zh. Gen. Khim.(USSR) 1990, 60, 2725. 